Supplementary Information

\title{
Splice Modulation Synergizes Cell Cycle Inhibition
}

Kelsey A. Trieger, ${ }^{\dagger}$ James J. La Clair, ${ }^{\dagger}$ and Michael D. Burkart ${ }^{\dagger}, *$

${ }^{\dagger}$ Department of Chemistry and Biochemistry, University of California, San Diego, La Jolla, CA 920930358, United States

*Correspondence: Michael D. Burkart, Phone: 858-534-5673, e-mail: mburkart@ucsd.edu

\section{Table of Contents}

Supplementary Figure S1

Supplementary Figure S2

Supplementary Figure S3

Supplementary Figure S4

Supplementary Table S1 S5

Supplementary Table S2 
<smiles>[R][R]=C=[R]=C</smiles>

FD-895 (1a) $\mathrm{R}_{1}=\mathrm{OH}, \mathrm{R}_{2}=\mathrm{CH}_{3}$ pladienolide $B(1 b) \quad R_{1}=H, R_{2}=H$<smiles>CO[C@@H]([C@H](C)[C@@](C)(O)C[C@@H](C)/C=C/C=C(\C)[C@@H](C)OC)[C@@H](C)O</smiles>

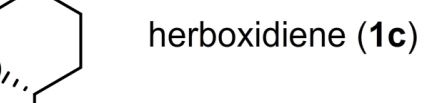

FR901464 (1d)<smiles>CC[C@@H]1C[C@H](P)[C@@H](C)O[C@@H]1CC=C(C)C=CC1O[C@](C)(O)C[C@@]2(CO2)[C@@H]1O</smiles>

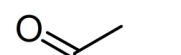

E7107 (1e)

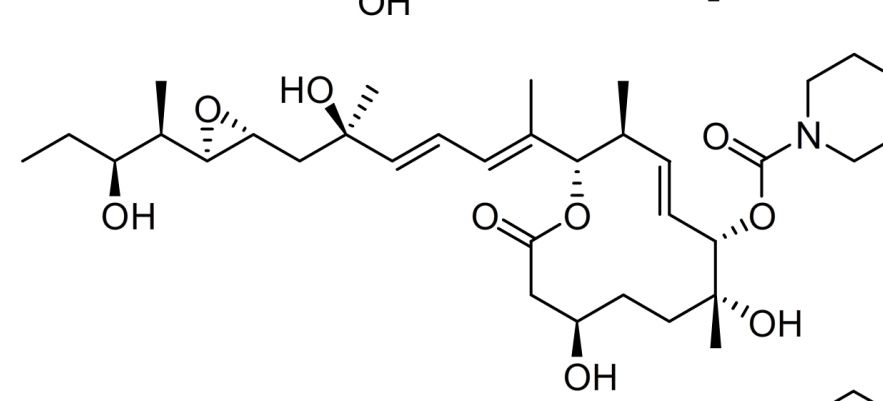

17S-FD-895 (1g) H3B-8800 (1f)<smiles>C/C(=C\C=C\[C@H](C)c1ccccn1)[C@H](O)OC(=O)C[C@H](O)CC[C@](C)(O)[C@H](/C=C/[C@@H](C)O)OC(=O)N1CCN(C)CC1</smiles>

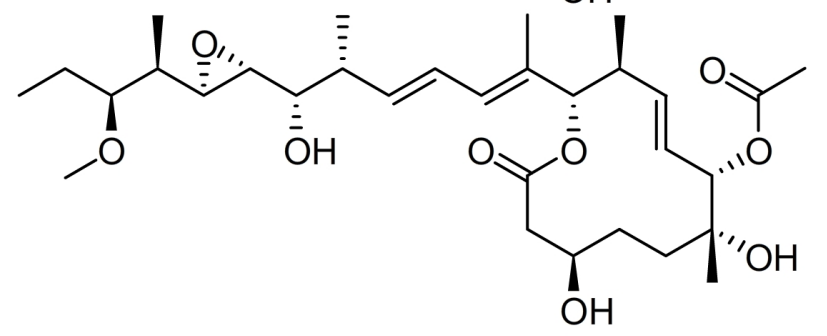

Supplementary Figure S1. Expanded set of splicing modulators including natural products FD-895 (1a), pladienolide B (1b), herboxidiene (1c), and FR901464 (1d). Structures of clinically-evaluated analogs E7107 (1e) and H3B8800 (1f) along with clinical-candidate 17S-FD-895 (1g). 


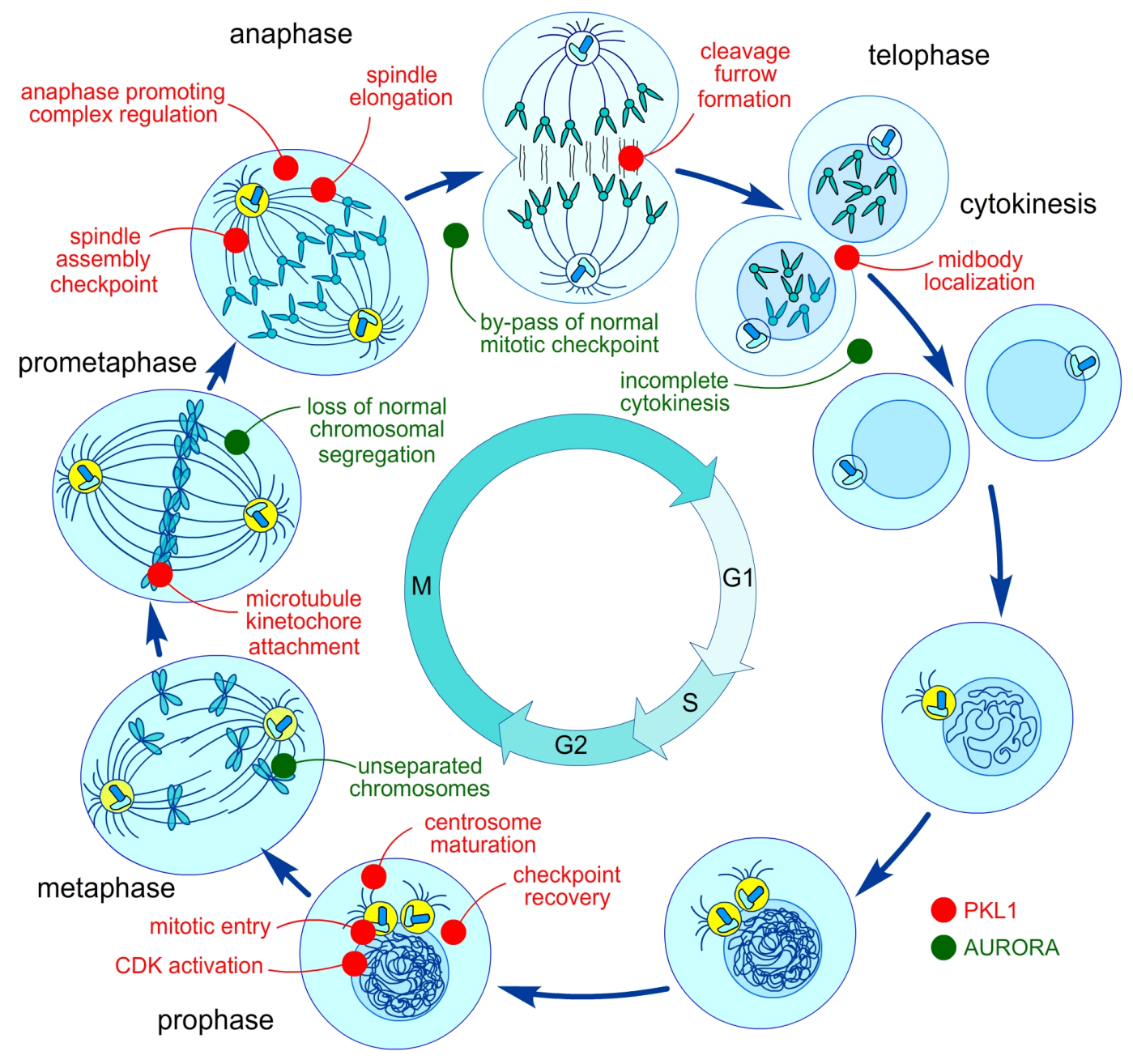

Supplementary Figure S2. Roles of polo-like kinase 1 (PLK-1) and aurora kinases (AURK) in the mitotic cycle. Both kinases play an interactive role in regulating each stage of passage through mitosis with effects arising from inhibition or reduced expression of PLK-1 (in red) and aurora kinases (in green). 


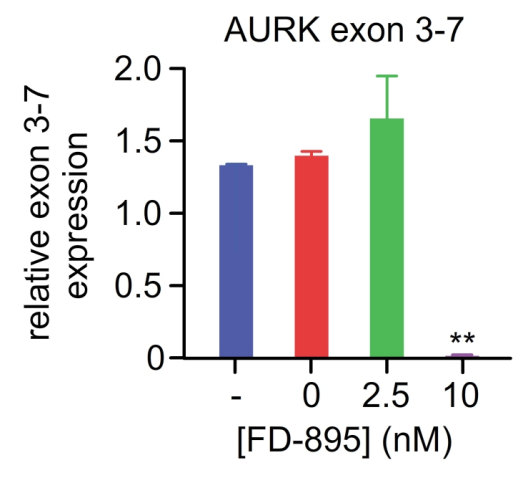

Supplementary Figure S3. AURKA gene regulation by FD-895. Increasing concentrations of FD-895 led to a decrease in the inclusion of exons 3-7 in AURKA RNA. 
a)

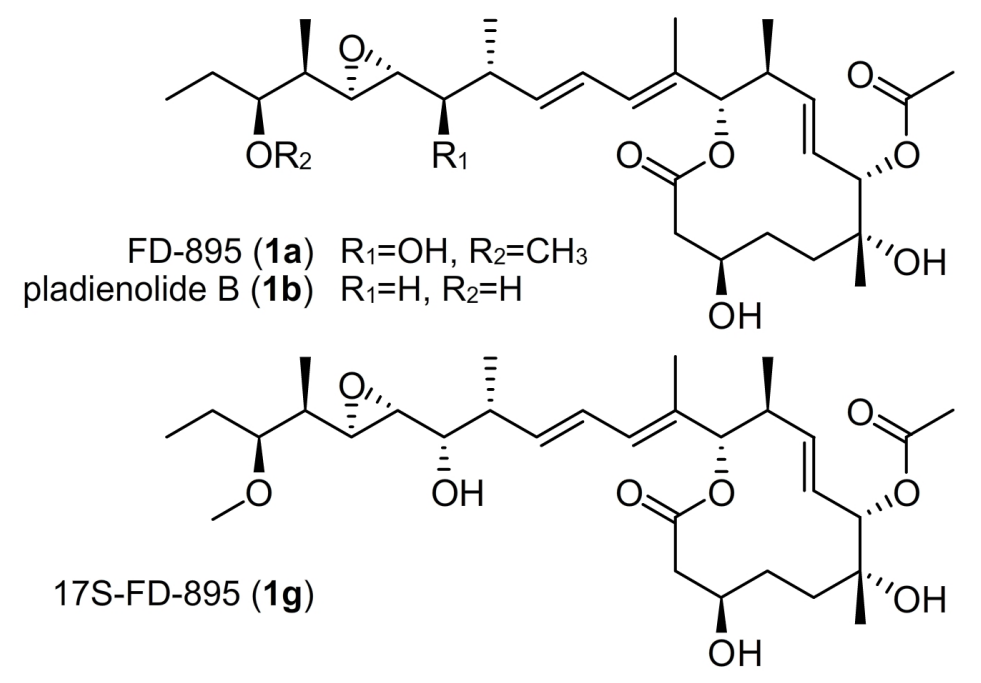

b)

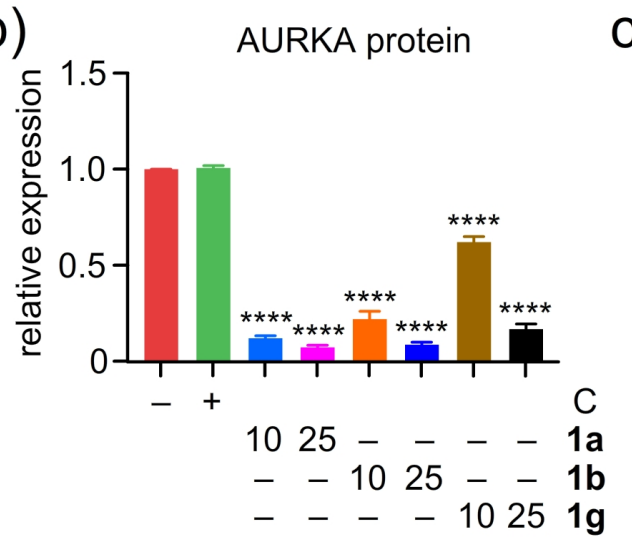

c)

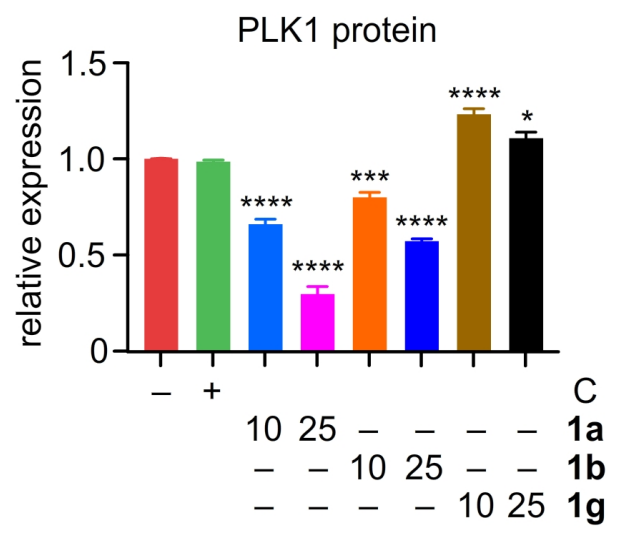

Supplementary Figure S4. Splice modulator selectivity. FD-895 (1a) and pladienolide B (1b) reduced AURKA and PLK-1 protein expression in a concentration-dependent manner. 17S-FD-895 (1g) reduced AURKA expression and modestly increased PLK-1 expression. Experiments were conducted in biological triplicate. Statistics were calculated using a standard one-way ANVOA; $\mathrm{p}$-values were represented so that $*$ signifies $\mathrm{p}<0.05, * *$ signifies $\mathrm{p}<0.01, * * *$ signifies $\mathrm{p}<0.001$, and $* * * *$ signifies $\mathrm{p}<0.0001$. 


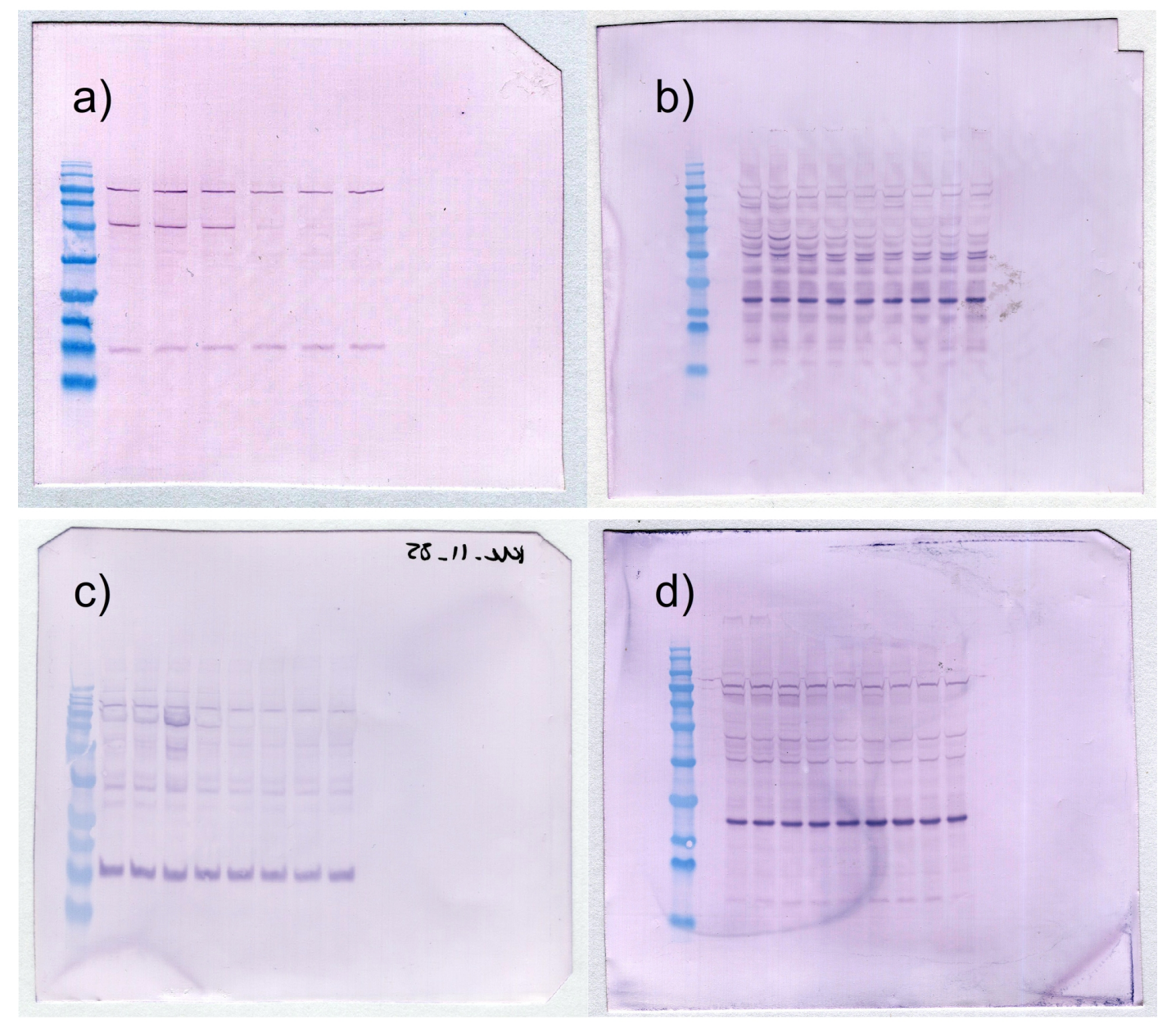

Supplementary Figure S5. Full scale scans of representative western blots used in this manuscript. a) Representative western blot used in Figure $3 \mathrm{~d}$. b) Representative western blot used in Figure 3e. c) Representative western blot used in Figure 4c. d) Representative western blot used in Figure 4d. 
Supplementary Table 1. Sequences of the qPCR primers used in this study.

\begin{tabular}{|c|c|c|}
\hline \multicolumn{2}{|c|}{ qPCR primers } \\
\hline Primer & Location & 5' Sequence 3' \\
\hline$G A P D H-\mathrm{FP}$ & Exon 3 & TGGTCACCAGGGCTGCTT \\
\hline$G A P D H-\mathrm{RP}$ & Exon 4 & AGCTTCCCGTTCTCAGCCTT \\
\hline$A U R K A-\mathrm{FP}$ & Intron 2 & CCACCTTCGGCATCCTAAT \\
\hline$A U R K A-\mathrm{RP}$ & Intron 2 & TCCAAGTGGTGCATATTCC \\
\hline$A U R K B-\mathrm{FP}$ & Intron 2 & GGAGAGCTTAAAATTGCAG \\
\hline$A U R K B-\mathrm{RP}$ & Intron 2 & TGCAGCTCTTCTGCAGCTC \\
\hline$P L K 1-\mathrm{FP}$ & & CTCAACACGCCTCATCCTC \\
\hline$P L K 1-\mathrm{RP}$ & & GTGCTCGCTCATGTAATTG \\
\hline \multicolumn{2}{|c}{} \\
\hline
\end{tabular}


Supplementary Table 2. Confidence intervals of $\mathrm{GI}_{50}$ values for cytotoxicity studies.*

\begin{tabular}{|c|c|c|c|c|}
\hline Compounds & Cell Line & $\mathrm{GI}_{50}$ value $(\mathrm{nM})$ & Standard error (nM) & Confidence interval \\
\hline $1 \mathrm{a}$ & HCT116 & $0.5-1.7$ & $0.1-0.4$ & $0.4-2.6$ \\
\hline $\mathbf{1 a}$ & Caov3 & $1.6-2.0$ & $0.46-0.59$ & $0.94-3.46$ \\
\hline $1 \mathbf{a}$ & HeLa & $3.7-4.5$ & $0.92-1.26$ & $2.16-8.24$ \\
\hline $2 \mathbf{a}$ & HCT116 & 430.0 & 440.7 & $3453.0-5231.0$ \\
\hline $\mathbf{2 a}+1 \mathrm{nM} \mathbf{1 a}$ & HCT116 & 517.0 & 281.0 & $136.9-1886.0$ \\
\hline $\mathbf{2 a}+5$ nM 1a & HCT116 & 0.05 & 0.05 & $0.01-0.18$ \\
\hline $2 \mathbf{a}$ & Caov3 & 5500 & 621 & $4303-6938$ \\
\hline $\mathbf{2 a}+1$ nM 1a & Caov3 & 2800 & 450 & $1906-3979$ \\
\hline $\mathbf{2 a}+5 \mathrm{nM} \mathbf{1 a}$ & Caov3 & 0.08 & 0.06 & $0.01-0.22$ \\
\hline $2 \mathbf{a}$ & HeLa & 10100 & 3371 & $5527-20150$ \\
\hline $\mathbf{2 a}+1 \mathrm{nM} \mathbf{1 a}$ & HeLa & 4000 & 1084 & $2368-6667$ \\
\hline $\mathbf{2 a}+5 \mathrm{nM} 1 \mathbf{a}$ & HeLa & 0.20 & 0.10 & $0.01-0.42$ \\
\hline $2 \mathbf{b}$ & HCT116 & 2100 & 186 & $1737-2492$ \\
\hline $\mathbf{2 b}+1 \mathrm{nM} \mathbf{1 a}$ & HCT116 & 250.0 & 35.7 & $180.3-351.6$ \\
\hline $\mathbf{2 b}+5 \mathrm{nM} \mathbf{1 a}$ & HCT116 & 0.05 & 0.04 & $0.01-0.13$ \\
\hline $2 \mathbf{b}$ & Caov3 & 6000 & 402 & $5233-6942$ \\
\hline $\mathbf{2 b}+1 \mathrm{nM} \mathbf{1 a}$ & Caov3 & 900 & 230 & $468-1765$ \\
\hline $\mathbf{2 b}+5 \mathrm{nM} \mathbf{1 a}$ & Caov3 & 0.07 & 0.06 & $0.01-0.19$ \\
\hline $2 \mathbf{b}$ & HeLa & 19500 & 4112 & $13180-31166$ \\
\hline $\mathbf{2 b}+1 \mathrm{nM} \mathbf{1 a}$ & HeLa & 16300 & 4178 & $10281-28836$ \\
\hline $\mathbf{2 b}+5 \mathrm{nM} \mathbf{1 a}$ & HeLa & 0.30 & 0.16 & $0.01-0.74$ \\
\hline $\mathbf{3 a}$ & HCT116 & 160.0 & 52.9 & $67.0-444.6$ \\
\hline $\mathbf{3 a}+1 \mathrm{nM} \mathbf{1 a}$ & HCT116 & 0.90 & 0.33 & $0.33-2.28$ \\
\hline $\mathbf{3 a}+5 \mathrm{nM} \mathbf{1 a}$ & HCT116 & 0.004 & 0.002 & $0.001-0.008$ \\
\hline $\mathbf{3 a}$ & Caov3 & 25.9 & 9.6 & $6.2-79.8$ \\
\hline $\mathbf{3 a}+1 \mathrm{nM} \mathbf{1 a}$ & Caov3 & 12.8 & 5.4 & $1.7-48.8$ \\
\hline $\mathbf{3 a}+5$ nM 1a & Caov3 & 0.20 & 0.05 & $0.07-0.30$ \\
\hline $\mathbf{3 a}$ & HeLa & 13200 & 2662 & $8927-20456$ \\
\hline $\mathbf{3 a}+1 \mathrm{nM} \mathbf{1 a}$ & HeLa & 10700 & 2369 & $7036-17217$ \\
\hline
\end{tabular}

* Data from these studies was obtained from and reported in Figure 2 
Supplementary Table 3. Combination Index values for cytotoxicity studies.*

\begin{tabular}{|c|c|c|c|c|c|}
\hline Figure & {$[\mathbf{1 a}](\mathrm{nM})$} & {$[\mathbf{2 a}](\mathrm{nM})$} & {$[2 \mathrm{~b}](\mathrm{nM})$} & {$[2 \mathrm{~b}](\mathrm{nM})$} & CI value \\
\hline \multirow{8}{*}{$2 a$} & 1.0 & 1.0 & & & 2.35 \\
\hline & 1.0 & 10.0 & & & 12.4 \\
\hline & 1.0 & 50.0 & & & 28.4 \\
\hline & 1.0 & 100.0 & & & 24.5 \\
\hline & 1.0 & 500.0 & & & 12.2 \\
\hline & 1.0 & $1,000.0$ & & & 4.34 \\
\hline & 1.0 & $5,000.0$ & & & 0.190 \\
\hline & 1.0 & $10,000.0$ & & & 0.166 \\
\hline \multirow{8}{*}{$2 a$} & 5.0 & 1.0 & & & 0.0605 \\
\hline & 5.0 & 10.0 & & & 0.0741 \\
\hline & 5.0 & 50.0 & & & 0.0525 \\
\hline & 5.0 & 100.0 & & & 0.0403 \\
\hline & 5.0 & 500.0 & & & 0.0530 \\
\hline & 5.0 & $1,000.0$ & & & 0.0681 \\
\hline & 5.0 & $5,000.0$ & & & 0.627 \\
\hline & 5.0 & $10,000.0$ & & & 0.833 \\
\hline \multirow{8}{*}{$2 b$} & 1.0 & 1.0 & & & $12,700,000$ \\
\hline & 1.0 & 10.0 & & & $76,600,000$ \\
\hline & 1.0 & 50.0 & & & 404,000 \\
\hline & 1.0 & 100.0 & & & 1,600 \\
\hline & 1.0 & 500.0 & & & $1,790,000$ \\
\hline & 1.0 & $1,000.0$ & & & 3,300 \\
\hline & 1.0 & $5,000.0$ & & & 10.7 \\
\hline & 1.0 & $10,000.0$ & & & 0.0962 \\
\hline \multirow{8}{*}{$2 b$} & 5.0 & 1.0 & & & 0.000218 \\
\hline & 5.0 & 10.0 & & & 0.000329 \\
\hline & 5.0 & 50.0 & & & 0.000348 \\
\hline & 5.0 & 100.0 & & & 0.000372 \\
\hline & 5.0 & 500.0 & & & 0.000567 \\
\hline & 5.0 & $1,000.0$ & & & 0.000810 \\
\hline & 5.0 & $5,000.0$ & & & 0.00275 \\
\hline & 5.0 & $10,000.0$ & & & 0.00519 \\
\hline \multirow{8}{*}{$2 \mathrm{c}$} & 1.0 & 1.0 & & & $498,000,000$ \\
\hline & 1.0 & 10.0 & & & 30,400 \\
\hline & 1.0 & 50.0 & & & $1,790,000$ \\
\hline & 1.0 & 100.0 & & & $1,790,000$ \\
\hline & 1.0 & 500.0 & & & $1,790,000$ \\
\hline & 1.0 & $1,000.0$ & & & $76,600,000$ \\
\hline & 1.0 & $5,000.0$ & & & 78.6 \\
\hline & 1.0 & $10,000.0$ & & & 0.0000407 \\
\hline \multirow{4}{*}{$2 c$} & 5.0 & 1.0 & & & 0.00811 \\
\hline & 5.0 & 10.0 & & & 0.00642 \\
\hline & 5.0 & 50.0 & & & 0.00642 \\
\hline & 5.0 & 100.0 & & & 0.0156 \\
\hline
\end{tabular}




\begin{tabular}{|c|c|c|c|c|}
\hline & 5.0 & 500.0 & & 0.0157 \\
\hline & 5.0 & $1,000.0$ & & 0.00234 \\
\hline & 5.0 & $5,000.0$ & & 0.0000237 \\
\hline & 5.0 & $10,000.0$ & & 0.0000108 \\
\hline \multirow{8}{*}{$2 d$} & 1.0 & & 1.0 & 4,580 \\
\hline & 1.0 & & 10.0 & 6.75 \\
\hline & 1.0 & & 50.0 & 2.56 \\
\hline & 1.0 & & 100.0 & 2.30 \\
\hline & 1.0 & & 500.0 & 0.519 \\
\hline & 1.0 & & $1,000.0$ & 0.268 \\
\hline & 1.0 & & $5,000.0$ & 0.260 \\
\hline & 1.0 & & $10,000.0$ & 0.0409 \\
\hline \multirow{8}{*}{$2 d$} & 5.0 & & 1.0 & 0.0761 \\
\hline & 5.0 & & 10.0 & 0.0823 \\
\hline & 5.0 & & 50.0 & 0.0686 \\
\hline & 5.0 & & 100.0 & 0.0787 \\
\hline & 5.0 & & 500.0 & 0.0769 \\
\hline & 5.0 & & $1,000.0$ & 0.0890 \\
\hline & 5.0 & & $5,000.0$ & 0.181 \\
\hline & 5.0 & & $10,000.0$ & 0.450 \\
\hline \multirow{8}{*}{$2 \mathrm{e}$} & 1.0 & & 1.0 & 587,000 \\
\hline & 1.0 & & 10.0 & 426,000 \\
\hline & 1.0 & & 50.0 & 154,000 \\
\hline & 1.0 & & 100.0 & 54,800 \\
\hline & 1.0 & & 500.0 & 2,090 \\
\hline & 1.0 & & $1,000.0$ & 223 \\
\hline & 1.0 & & $5,000.0$ & 8.74 \\
\hline & 1.0 & & $10,000.0$ & 4.04 \\
\hline \multirow{8}{*}{$2 \mathrm{e}$} & 5.0 & & 1.0 & 0.00242 \\
\hline & 5.0 & & 10.0 & 0.01263 \\
\hline & 5.0 & & 50.0 & 0.00593 \\
\hline & 5.0 & & 100.0 & 0.00882 \\
\hline & 5.0 & & 500.0 & 0.0251 \\
\hline & 5.0 & & $1,000.0$ & 0.0187 \\
\hline & 5.0 & & $5,000.0$ & 0.0123 \\
\hline & 5.0 & & $10,000.0$ & 0.0120 \\
\hline \multirow{8}{*}{$2 \mathrm{f}$} & 1.0 & & 1.0 & $307,000,000,000$ \\
\hline & 1.0 & & 10.0 & $307,000,000,000$. \\
\hline & 1.0 & & 50.0 & $307,000,000,000$. \\
\hline & 1.0 & & 100.0 & $307,000,000,000$. \\
\hline & 1.0 & & 500.0 & $1,108,000,000$ \\
\hline & 1.0 & & $1,000.0$ & $307,000,000,000$. \\
\hline & 1.0 & & $5,000.0$ & $1,108,000,000$ \\
\hline & 1.0 & & $10,000.0$ & 8.53 \\
\hline \multirow{3}{*}{$2 \mathrm{f}$} & 5.0 & & 1.0 & 0.0591 \\
\hline & 5.0 & & 10.0 & 0.0344 \\
\hline & 5.0 & & 50.0 & 0.437 \\
\hline
\end{tabular}




\begin{tabular}{|c|c|c|c|c|}
\hline & 5.0 & 100.0 & & 0.135 \\
\hline & 5.0 & 500.0 & & 0.0156 \\
\hline & 5.0 & $1,000.0$ & & 0.0156 \\
\hline & 5.0 & $5,000.0$ & & 0.000324 \\
\hline & 5.0 & $10,000.0$ & & 0.0000536 \\
\hline \multirow{8}{*}{$2 \mathrm{~g}$} & 1.0 & & 1.0 & 4.03 \\
\hline & 1.0 & & 10.0 & 1.57 \\
\hline & 1.0 & & 50.0 & 1.49 \\
\hline & 1.0 & & 100.0 & 1.66 \\
\hline & 1.0 & & 500.0 & 0.807 \\
\hline & 1.0 & & $1,000.0$ & 0.501 \\
\hline & 1.0 & & $5,000.0$ & 0.00378 \\
\hline & 1.0 & & $10,000.0$ & 0.000414 \\
\hline \multirow{8}{*}{$2 \mathrm{~g}$} & 5.0 & & 1.0 & 0.0254 \\
\hline & 5.0 & & 10.0 & 0.00704 \\
\hline & 5.0 & & 50.0 & 0.00415 \\
\hline & 5.0 & & 100.0 & 0.00712 \\
\hline & 5.0 & & 500.0 & 0.0185 \\
\hline & 5.0 & & $1,000.0$ & 0.00472 \\
\hline & 5.0 & & $5,000.0$ & 0.0170 \\
\hline & 5.0 & & $10,000.0$ & 0.0000336 \\
\hline \multirow{8}{*}{$2 \mathrm{~h}$} & 1.0 & & 1.0 & $35,700,000$ \\
\hline & 1.0 & & 10.0 & $240,000,000$ \\
\hline & 1.0 & & 50.0 & 922,000 \\
\hline & 1.0 & & 100.0 & $4,470,000$ \\
\hline & 1.0 & & 500.0 & 2,610 \\
\hline & 1.0 & & $1,000.0$ & 4,330 \\
\hline & 1.0 & & $5,000.0$ & 10.4 \\
\hline & 1.0 & & $10,000.0$ & 0.0938 \\
\hline \multirow{8}{*}{$2 \mathrm{~h}$} & 5.0 & & 1.0 & 0.000188 \\
\hline & 5.0 & & 10.0 & 0.000412 \\
\hline & 5.0 & & 50.0 & 0.000309 \\
\hline & 5.0 & & 100.0 & 0.000295 \\
\hline & 5.0 & & 500.0 & 0.000447 \\
\hline & 5.0 & & $1,000.0$ & 0.000879 \\
\hline & 5.0 & & $5,000.0$ & 0.00385 \\
\hline & 5.0 & & $10,000.0$ & 0.00455 \\
\hline \multirow{8}{*}{$2 \mathrm{i}$} & 1.0 & & 1.0 & $307,000,000,000$ \\
\hline & 1.0 & & 10.0 & $6,140,000$ \\
\hline & 1.0 & & 50.0 & $76,600,000$ \\
\hline & 1.0 & & 100.0 & $76,600,000$ \\
\hline & 1.0 & & 500.0 & $1,790,000$ \\
\hline & 1.0 & & $1,000.0$ & $307,000,000,000$ \\
\hline & 1.0 & & $5,000.0$ & 144,000 \\
\hline & 1.0 & & $10,000.0$ & 1.59 \\
\hline \multirow{2}{*}{$2 \mathrm{i}$} & 5.0 & & 1.0 & 0.0156 \\
\hline & 5.0 & & 10.0 & 0.0234 \\
\hline
\end{tabular}




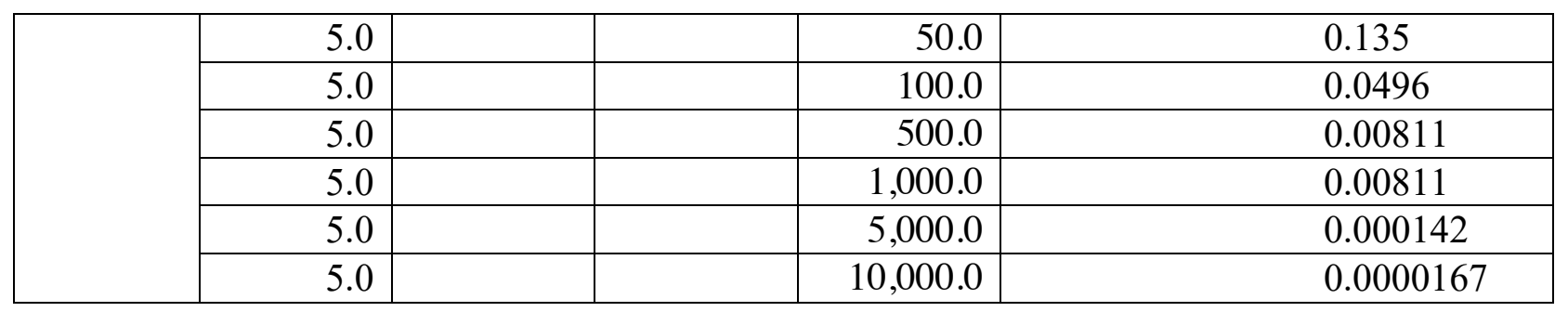

*Data from these studies was obtained from and reported in Figure 2 